\title{
$\mathcal{A}$ Interferência do Sistema Consonantal Português no Uso do Espanhol
}

\section{La Interferencia del Sistema Consonántico Portugués en el USO DEL ESPAÑOL}

\section{Luciene Bassols BRISOLARA *}

Resumo: Este artigo discute a necessidade do ensino da pronúncia do espanhol como língua estrangeira, considerando que, dentre os vários aspectos de uma língua, as atividades relacionadas à pronúncia costumam receber pouca atenção em sala de aula. Para desenvolver esta pesquisa, analisamos amostras de fala de estudantes do Curso de Letras, português/espanhol, da Universidade Federal do Rio Grande, em fase de aquisição do espanhol, buscando descrever e examinar a interlíngua fônica dos estudantes, e também propor procedimentos metodológicos para corrigir as interferências relativas à pronúncia. Os resultados deste estudo demonstraram que, em sala de aula, é necessário que sejam propostas atividades regulares que estimulem a correção fonética, o que proporcionará o desenvolvimento da competência fônica na língua meta.

Palavras-chave: Interferência fônica. Aquisição de espanhol como LE. Ensino de pronúncia.

Resumen: Esta investigación discute la necesidad de la enseñanza de la pronunciación del español como LE, considerando que, entre los varios aspectos de una lengua, las actividades relacionadas a la pronunciación suelen recibir poca atención en el aula. Para desarrollar

\footnotetext{
* Profesora de lengua española y lingüística aplicada a la enseñanza de español como lengua extranjera, del Instituto de Letras e Artes de la Universidade Federal do Rio Grande (FURG); responsable por el proyecto de investigación 'Interferencias fonético/fonológicas en la adquisición del español por hablantes nativos de portugués'. Contato: lucienebrisolara@furg.br
} 
el estudio, analizamos muestras de habla de estudiantes del Curso de Letras, portugués/español, en la FURG en fase de adquisición del español, buscando describir y examinar la interlengua fónica de los estudiantes, y también proponer procedimientos metodológicos para corregir las interferencias relativas a la pronunciación.

Palabras clave: Interferencia fónica. Adquisición del español como LE. Enseñanza de la pronunciación

\section{Introducción}

Uno de los problemas que plantea la enseñanza del español a brasileños es la semejanza existente en algunos aspectos lingüísticos entre esos idiomas. En el léxico, por ejemplo, hay unas cuantas palabras que se escriben igual y que, en muchos casos, presentan el mismo significado. Por otro lado, en el campo de la pronunciación, gran parte de estas palabras suena diferente en las dos lenguas. De ahí que surgen los problemas en el aprendizaje de esos idiomas como lengua extranjera ${ }^{1}$. A pesar de que el brasileño pueda comunicarse en español en el primer contacto con esta lengua, surgen muchas formas que no integran el español, las cuales suelen constituir transferencias de su lengua materna.

El estudiante brasileño presenta un imaginario de que el español es una lengua fácil, transparente, dado que al viajar a Uruguay, Argentina, etc., es capaz de comprender relativamente a los hablantes nativos de ese idioma. A partir de esa visión, el alumno suele no prestar atención en los aspectos fonético/fonológicos, morfológicos, sintácticos, semánticos y discursivos de la lengua. Así, pasa mucho tiempo usando la interlengua y eso preocupa al profesor, ya que esas formas no nativas pueden llegar al nivel de la fosilización. En este nivel los estudiantes mantienen en su interlengua rasgos que no forman parte de la lengua meta, como aspectos gramaticales, léxicos, pronunciaciones equivocadas, etc., alejándose de los usuarios naturales de la lengua española.

${ }^{1}$ En este estudio consideramos los términos 'lengua extranjera', 'lengua segunda' o 'segunda lengua' como sinónimos, es decir, una lengua que se aprende sea en contexto formal o natural, diferente de la lengua materna. 
Según Corder (1967, p. 11):

los errores son indispensables para el aprendiz, pues se puede considerar el error como un procedimiento utilizado por el aprendiz para aprender. Es un medio de verificar sus hipótesis sobre el funcionamiento de la lengua que aprende. Cometer errores, es pues una estrategia que emplean los niños en la adquisición de su lengua materna y también los aprendices de una L2.

En este estudio, por lo tanto, consideramos que el proceso de interlengua integra el aprendizaje y que el alumno necesita equivocarse para aprender el nuevo idioma. Cabe al profesor ayudar al estudiante en la superación de sus dificultades, estimulándolo a repensar o confirmar sus hipótesis sobre el funcionamiento de la LE.

Para analizar la interlengua fónica del aprendiz en esta investigación nos proponemos a verificar cómo se realiza la adquisición del sistema consonántico del español por brasileños. El objetivo del estudio es verificar si los académicos de Letras (Portugués/Español) de la Universidade Federal do Rio Grande al aprender español emplean las consonantes de este idioma o si sufren interferencias fonéticas del portugués en lo que se refiera a los siguientes procesos: palatalización de las oclusivas dentales; vocalización de / $/$ en posición de coda; diptongación de la nasal en final de palabra y sonorización de /s/ intervocálica.

\section{Español y Portugués, parecido no es lo mismo: las trampas existentes en los aspectos fonético/fonológicos de esos idiomas}

A pesar de que el español y el portugués sean lenguas próximas, existen muchas diferencias con relación a los aspectos fónicos de esos idiomas. Las principales diferencias entre esos sistemas lingüísticos se presentan en el cuadro a continuación y tienen como base los estudios de Câmara Jr. (1970), Hora (2000), Espiga (2001), Brandão (2003), Mena-Alves y Brisolara (2004). 
a) Siete fonemas vocálicos: b[i]co, $\mathrm{b}[\mathrm{e}] \mathrm{co}, \mathrm{b}[\mathrm{\varepsilon}] \mathrm{la}, \mathrm{b}[\mathrm{a}] \mathrm{la}, \mathrm{b}[\mathrm{o}] \mathrm{lo}, \mathrm{b}[\mathrm{o}] \mathrm{la}$, $\mathrm{b}[\mathrm{u}] \mathrm{la}$;

b) Las vocales varían según el acento y su posición en la palabra: pretónica: $\mathrm{f}[\varepsilon] \mathrm{rro} \rightarrow \mathrm{f}[\mathrm{e}]$ rreiro; $\mathrm{p}[\mathrm{\rho}] \mathrm{rta}$ $\rightarrow$ p[o]rteiro; postónica no final: abóbora $\rightarrow$ abób[u]ra; fósforo $\rightarrow$ fósf[u]ro; postónica final: ovo $\rightarrow$ ov[u]; leque $\rightarrow$ lequ[i];

c) Las vocales pueden nasalizarse siempre que haya una consonante nasal próxima a ellas: $\operatorname{manh}[\tilde{a}]$, [ã]ntes, am[ã]nte, c[ã]ntar, etc;

d) Los fonemas vocálicos /e/ /o/, /i/ y /u/ pueden convertirse en semivocales: $\mathrm{m}[\mathrm{j} a] \mathrm{mou}$ - 'me amou', mág[wa] - 'magoa', 1[ej]te - 'leite', s[ow] - 'sou';

e) Fonemas del portugués que constituyen alófonos del español: $/ \int / \mathrm{y} / 3 / \mathrm{a}$ a[S]o - 'acho' $\neq \mathrm{a}[3] \mathrm{o}-$ 'ajo', en portugués; ca[J]e $\sim$ ca[3]e 'calle', en español;

f) Procesos consonánticos exclusivos del portugués: sonorización de /s/ en posición intervocálica: me $[\mathrm{z}] \mathrm{a}-$ 'mesa', ca[z]a - 'casa'; vocalización de /1/ en posición de coda: pape[w] - 'papel', jorna[w] - 'jornal'; diptongación de la consonante nasal en posición final de palabra:

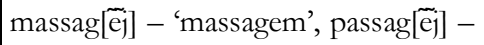
'passagem'; palatalización de /t/ y /d/ ante la vocal [i]: [t $\left.\int\right]$ ia - 'tia', [d3]ia - 'dia';

g) Estructura silábica: inserción de [i] epentético ante consonantes en posición final de sílaba: a[pi]to 'apto', o[bi]strução - 'obstrução', e[t]i]nia - 'etnia', a[d]3i]vogado 'advogado', i[gi]norante - a) Cinco fonemas vocálicos: p[i]so, $\mathrm{p}[\mathrm{e}] \mathrm{so}, \mathrm{p}[\mathrm{a}] \mathrm{so}, \mathrm{p}[\mathrm{o}] \mathrm{so}, \mathrm{p}[\mathrm{u}] \mathrm{so}$;

b) Las vocales se mantienen en cualquier posición en la palabra: $\mathrm{h}[\mathrm{o}] \mathrm{mbre}$; $\mathrm{h}[\mathrm{o}] \mathrm{rmiga}$; niñ $[\mathrm{o}]$; b[e]llo; r[e]galo; part[e];

c) Las vocales sólo sufren nasalización cuando se encuentran entre dos consonantes nasales o cuando aparecen después de pausa y antes de una consonante nasal: am[ã]nte, [ã]ntes. En los demás casos la vocal se produce sin nasalidad, es decir, como vocal oral: c[a]ntar, jueg[a]n;

d) A diferencia del portugués, los únicos fonemas que pueden convertirse en semivocales son /i/ y /u/: f[je]sta - 'fiesta', p[ein]ne 'peine', b[we]no - 'bueno', b[oun] 'bou';

e) Fonemas del español que constituyen alófonos del portugués: $/ \mathrm{t}] /:[\mathrm{t}]]$ ino - 'chino' $\neq[\mathrm{t}]$ ino 'tino', en español; [t]]ia $\sim$ [t]ia - 'tia', en portugués;

f) Procesos consonánticos exclusivos del español: fricativización de las consonantes oclusivas: ca[ß] - 'cava' na[ð]a - 'nada' a[r]ua - 'agua'; neutralización de las oclusivas en posición de coda: $\mathrm{a}[\mathrm{k}]$ to $\sim \mathrm{a}[\mathrm{g}]$ to $~$ a[r]to - 'acto' ; seseo: ca[s]ar 'casar' ca[s]ar - 'cazar'; yeísmo: ca[J]o; ca[3]o - 'cayo';

g) Estructura silábica: la consonante en posición final de sílaba se mantiene sin que se inserte la vocal [i]: ó[p]timo - 'óptimo', o[b]turar 'obturar', é[t]nico - 'étnico', ciuda[d] - 'ciudad', a[k]to - acto', i[g]norante - 'ignorante'. 
Como se puede ver, el portugués y el español presentan diferencias significativas en lo referente a los aspectos fonético/ fonológicos y aunque estos idiomas posean semejanzas, existen varias particularidades no sólo fonético/fonológicas sino también morfológicas, sintácticas y semánticas entre ellos. Esos desencuentros entre esas lenguas hacen que el hablante nativo de portugués, al aprender español, produzca una serie de interferencias de su LM en la LE. Las interferencias de naturaleza fonético/fonológicas son bastante frecuentes en la adquisición del español como LE, de modo especial y con características específicas por hablantes de lengua portuguesa.

En ese sentido, este trabajo tratará de analizar algunos procesos de interferencias de la LM, en este caso el portugués, en la adquisición del español. Obsérvese que en el punto 2.1 presentamos los procesos fonético/fonológicos que serán investigados en este estudio.

\subsection{Procesos de interferencias fonético/fonológicas del portugués en el aprendizaje de español}

A continuación describiremos cuatro procesos característicos del portugués que analizaremos en esta investigación. Vale destacar que todos son fenómenos prácticamente categóricos en el portugués hablado en el sur de Brasil.

Las consonantes $/ \mathrm{t} / \mathrm{y} / \mathrm{d} /$, en portugués, se producen como oclusivas alveolares o dentales, siempre que anteceden las vocales [a],

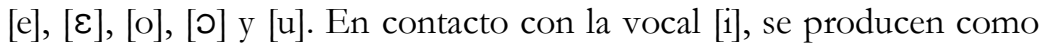
africadas, y ese fenómeno se denomina palatalización de $/ t / y / d /^{2}$. El resultado es el alófono [t] para el fonema /t/ y [d3] para el fonema / $\mathrm{d} /$. Como ejemplo tenemos den [t $\mathrm{T} i$ sta - 'dentista' y [d3i]vórcio ‘divórcio’. La palatalización también sucede cuando las consonantes /t/ y /d/ están en posición de coda silábica, porque en portugués hay una epéntesis de [i] después de esas consonantes. Como ejemplos tenemos: a[dzi]mirar - 'admirar' y a tt i] mosfera - 'atmosfera'.

2 Para otras informaciones sobre el proceso de palatalización en portugués ver Hora (1990), Sassi (1997), Carvalho (1999), Brisolara, Vandresen y Matzenauer (2002). 
Vale destacar que la palatalización de esas oclusivas no es un fenómeno existente en español y que el alófono del portugués [t $\left.\int\right]$ tiene estatus de fonema en español; en otras palabras, el cambio de $[t]$ por $\left[\mathrm{t} \int\right]$ en este idioma puede implicar en el cambio de significado. Como

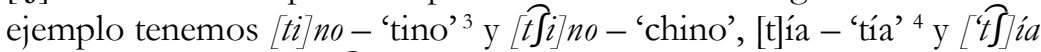
- 'chía', [t]ic - 'tic' y [t]]ic- 'chic'.

Según Navarro Tomás (2004), en español /t/ en posición de ataque se produce como oclusiva dental independiente de la vocal que anteceda. Por otro lado, /d/ se produce como oclusiva o fricativa dental; si viene después de una consonante nasal, de la aproximante lateral /1/ o está en inicio absoluto, se produce como [d], en los demás entornos se produce como [ð]. En posición de coda no hay epéntesis de la vocal [i], pero ambas consonantes suelen producirse de manera débil y relajada, siendo el resultado [ð].

Otra consonante que en portugués se produce diferente del español es $/ 1 /$. En posición de ataque simple o complejo en ambas lenguas el resultado fonético es igual, es decir aproximante lateral alveolar, como vemos en las palabras [l]entes - 'lentes', ca[l]or - 'calor', b[l]usa- 'blusa'.

Por otro lado, en posición de coda, español y portugués presentan diferencias fonéticas. En portugués el fonema /1/ se transforma en la semivocal $[\mathrm{w}]$, independiente si está en posición de coda interna o final; por ejemplo, $c a[w] m a$ - 'calma', nata $[w]$ - 'natal', norma [w] mente 'normalmente'. Ese proceso se denomina vocalización de /l/ en posición de coda.

${ }^{3}$ Tino: juicio y cordura; chino: natural de China (RAE. Disponible en www.rae.es)

${ }^{4}$ Tía: respecto de una persona, hermano o hermana de su padre o madre; Chía: a) manto negro y corto, regularmente de bayeta, que se ponía sobre el capuz y cubría hasta la mano, usado en los lutos antiguos; b) semilla de una especie de salvia. Remojada en agua, suelta gran cantidad de mucilago, que, con azúcar y zumo de limón, es un refresco muy usado en México. Molida, produce un aceite secante. (RAE. Disponible en www.rae.es)

${ }^{5}$ Tic: movimiento convulsivo, que se repite con frecuencia, producido por la contracción involuntaria de uno o varios músculos; Chic: elegante, distinguido, a la moda. (RAE. Disponible en www.rae.es) 
A diferencia del portugués, la lengua española no presenta vocalización de /1/. En este idioma la aproximante lateral en coda final, seguida de pausa o de consonante alveolar, se produce como alveolar; como ejemplo tenemos: ma[l] - 'mal', igua [l] - 'igual', natura[l] - 'natural', pu[l]so - 'pulso'. Si hay otra consonante después de /1/, la aproximante lateral asimila el punto de articulación de la consonante siguiente; por ejemplo, a[] to - 'alto' (asimilación de la consonante dental), co [l] cha - 'colcha' (asimilación de la consonante palatal), ca[l] zar - 'calzar' (asimilación de la consonante interdental).

En portugués, la consonante nasal, cuando en posición final de palabra, se realiza como semivocal, formando con la vocal antecedente, un diptongo, proceso denominado diptongación de la nasal en final de palabra. Como ejemplo tenemos las siguientes palabras: $n u v[\tilde{e j}]$ - 'nuvem', $b[\tilde{e j}]$ - 'bem', b[ow] - 'bom' y bomb[ow] - 'bombom'. En los ejemplos presentados arriba observamos que ocurre la producción del diptongo. En este caso, hay una doble asimilación: la vocal asimila la nasalidad de la consonante siguiente y esta asimila el punto de articulación de la vocal, creando una semivocal homorgánica.

La diptongación de la consonante nasal en final de palabra no ocurre en español y, según Alarcos (1991), la nasal en final de palabra, seguida de pausa, se produce como alveolar, como se puede verificar en los ejemplos presentados a continuación: bombó [n] - 'bombón', do [n] - 'don', folletí [n] - 'folletín' y fi $[n]$ - 'fin'.

Si la nasal está en coda interna sufre asimilación del punto de articulación de la consonante siguiente; por ejemplo, a[m]fiteatro 'anfiteatro' (asimilación de la consonante labiodental), die [n]te - 'diente' (asimilación de la consonante dental), ta[n]go - 'tango' (asimilación de la consonante velar), cha $\left.{ }^{j} n^{j}\right] c h o$ - 'chancho' (asimilación de la consonante palatal) y $u[m]$ beso - 'un beso' (asimilación de la consonante bilabial).

Una importante diferencia entre el español y el portugués es el hecho de que /z/ es un fonema en portugués y no en español: [z]inco - 'zinco' $\neq$ [s]inco - 'cinco'. Los ejemplos demuestran que /s/ y / z/ son fonemas consonánticos del portugués.

En español, sin embargo, existe sólo el fonema /s/, y éste tiene la forma $[z]$ como su alófono, en posición de coda, o sea, /s/ se produce como $[\mathrm{z}]$ ante una consonante sonora, dado que la sonoridad 
de esta consonante se transmite a la fricativa, como vemos en 'mu[z]lo' y 'de[z]de'.

$\mathrm{Al}$ adquirir el español, un hablante nativo de portugués suele producir [z] aun en posición de onset en ambiente intervocálico, ya que en portugués hay un fenómeno típico que se denomina sonorización de /s/ intervocálico; como ejemplo tenemos 'me[z]a' y 'ca[z]a' cuando en español se produciría 'me[s]a' y 'ca[s]a'.

En resumen, todos esos procesos descritos en este apartado constituyen características fonéticas del portugués, pero no del español; por era razón, fueron investigados en la producción oral de los alumnos del Curso de Letras, portugués/español de la Universidade Federal do Rio Grande y los resultados de los análisis serán presentados en el ítem 4. El objetivo de esta investigación es encontrar soluciones que faciliten la adquisición del español como LE, disminuyendo el grado de interferencia de la LM en la LE.

\section{Metodología}

Para la realización de este estudio se seleccionaron cuarenta entrevistas de estudiantes brasileños del curso de Profesorado en Letras (Portugués/ Español). Las entrevistas contemplan alumnos de los cuatro años del profesorado y en los dos turnos en el que se dicta, diurno y nocturno. La conversación que duraba aproximadamente 15 minutos ocurrió de manera libre y natural en la que los alumnos podían usar el conocimiento que tenían en manos de la lengua española para responder a las preguntas que se les hacían.

Las preguntas de la entrevista no eran complejas sin embargo exigían que el alumno tuviera un mínimo de conocimiento para que consiguiera responderlas de una manera coherente con el año que cursaba.

Por medio de estas informaciones haremos una comparación entre los cuatro años de estudio de E/LE para verificar de alguna

${ }^{6}$ Cabe destacar que la sonorización de /s/ en posición de coda ocurre en algunas regiones de Hispanoamérica, como, por ejemplo, en México. En otras zonas como en Uruguay se suele aspirar o apagar la fricativa /s/. 
manera dónde se encuentran las mayores dificultades de los alumnos respecto a la producción de las consonantes del español. De esa forma se desea contribuir para que la enseñanza del E/LE venga a perfeccionarse cada vez más y así se alcance el gran objetivo de capacitar a profesores que puedan ejercer su profesión con mayor seguridad y dominio a la hora de enseñar.

\section{Análisis de los datos y presentación de los resultados}

Según presentamos anteriormente, este estudio se constituye de entrevistas realizadas a académicos del Curso de Letras (Portugués/ Español) que cursan el primero, segundo, tercero y cuarto años de este profesorado. En las Tablas 1, 2, 3, 4 y 5, presentamos los resultados referentes a los cuatro procesos investigados en este estudio: palatalización de /t/ y /d/ ante [i], vocalización de /1/ en posición de coda, diptongación de la consonante nasal en final de palabra y sonorización de /s/ intervocálico.

Tabla 1: Palatalización de /t/ y /d/ ante [i]

\begin{tabular}{|l|c|c|c|}
\hline Escolaridad & $\begin{array}{c}\text { Palatalización de } \\
\text { /t/ y /d/ ante [i] / } \\
\text { Total de posibilidades }\end{array}$ & Porcentaje & Ejemplos \\
\hline Primero & $15 / 339$ & $4,42 \%$ & [no't]isja] \\
\hline Segundo & $19 / 299$ & $6,35 \%$ & [dzi'fisil] \\
\hline Tercero & $23 / 486$ & $4,73 \%$ & [lin'gwist]ika] \\
\hline Cuarto & $12 / 406$ & $2,95 \%$ & [estudzjá're] \\
\hline Total & $69 / 1.530$ & $4,50 \%$ & \\
\hline
\end{tabular}

Los resultados de la Tabla 1 indican que la palatalización presenta un índice pequeño de aplicación en las muestras representativas de todos los niveles de escolaridad, a pesar de que se la evidencie en los cuatro años del curso de español.

Es probable que el pequeño empleo de este proceso de interferencia de la Lengua Materna se justifique porque los estudiantes 
de español saben que $\longdiv { \mathrm { t } \int } /$ tiene estatus de fonema en español, y, no, de alófono de /t/, como ocurre en portugués. En la Tabla 2, presentamos separadamente los resultados de la palatalización de /t/ y /d/.

Tabla 2: Resultados estadísticos separados de la palatalización de $/ \mathrm{t} / \mathrm{y}$ de $/ \mathrm{d} /$ ante [i]

\begin{tabular}{|l|c|c|c|c|}
\hline Escolaridad & $\begin{array}{c}\text { Palatalización de } \\
\text { /t/ ante [i] / } \\
\text { Total de } \\
\text { posibilidades }\end{array}$ & Porcentaje & $\begin{array}{c}\text { Palatalización de } \\
\text { /d/ ante [i] / } \\
\text { Total de } \\
\text { posibilidades }\end{array}$ & Porcentaje \\
\hline Primero & $8 / 166$ & $4,81 \%$ & $7 / 173$ & $4,04 \%$ \\
\hline Segundo & $12 / 172$ & $6,97 \%$ & $7 / 127$ & $5,51 \%$ \\
\hline Tercero & $20 / 263$ & $7,60 \%$ & $3 / 223$ & $1,34 \%$ \\
\hline Cuarto & $9 / 200$ & $4,5 \%$ & $3 / 206$ & $1,45 \%$ \\
\hline Total & $49 / 801$ & $6,10 \%$ & $20 / 729$ & $2,74 \%$ \\
\hline
\end{tabular}

Al comparar los resultados de la palatalización de la oclusiva dental sorda con la sonora, notamos que la frecuencia del fenómeno de transferencia de la LM es mayor cuando la oclusiva es /t/ que cuando es /d/. En las entrevistas verificamos un aparecimiento ligeramente inferior de palabras con la dental sonora, que se puede verificar en los resultados totales de esta tabla, es decir, 801 datos de /t/ y 729, de /d/.

Sin embargo, cabe tener en cuenta que a pesar de que este proceso de transferencia del portugués no presente altos índices, permanece en el habla de los estudiantes en los cuatro anos de estudio, lo que puede fosilizarse, dificultando el perfeccionamiento en la lengua meta. 
Tabla 3: Vocalización de /1/ en posición de coda

\begin{tabular}{|l|c|c|c|}
\hline Escolaridad & $\begin{array}{c}\text { Vocalización de /1/ en } \\
\text { coda / Total de } \\
\text { posibilidades }\end{array}$ & Porcentaje & Ejemplos \\
\hline Primero & $60 / 276$ & $22 \%$ & [difikuún'tad] \\
\hline Segundo & $29 / 342$ & $8,5 \%$ & [profesionaun'mẽnnte] \\
\hline Tercero & $32 / 562$ & $5,7 \%$ & [kuưn'tura] \\
\hline Cuarto & $45 / 429$ & $10,5 \%$ & [espa'noun] \\
\hline Total & $166 / 1.609$ & $10,3 \%$ & \\
\hline
\end{tabular}

Conforme observamos en la Tabla 3, el proceso de vocalización presenta mayor incidencia en la muestra de primer año de escolaridad, presentando el $22 \%$ de ocurrencia. Por otro lado, en el segundo y en el tercer año, hay una significativa disminución de este tipo de transferencia fonética (el 8,5\% y el 5,7\%, respectivamente). Con todo, verificamos que hay un aumento de la vocalización en los datos relativos al cuarto año, o sea, el 10,5\%.

Vale destacar que los resultados relativos al proceso de vocalización indican que hay una preferencia por parte de los hablantes de emplear ese fenómeno cuando / 1 / está en el interior de palabra, es decir, coda medial. Del total de 60 casos de vocalización encontrados en el primer año, 49 eran de coda medial; en el segundo año, de los 29 datos, 26 presentaban coda medial; en el tercer año, todos los 32 datos de vocalización eran de coda medial; y en el cuarto, de los 45 casos de este fenómeno, en 42 casos la coda era medial.

Considerando los datos de conservación de la variante española, o sea, en que no hubo vocalización, encontramos un índice de aproximadamente el $40 \%$ de elementos monosilábicos en cada año de escolaridad. En el primer año, hubo un 33\% de monosílabos; en el segundo, un $37 \%$, en el tercero, un $41 \%$ y, en el cuarto, un $44 \%$. En estos casos, creemos que el mantenimiento de / / en coda se relaciona con el tamaño de la palabra, lo que puede facilitar el cuidado con la pronunciación adecuada. 
Tabla 4: Diptongación de la nasal en final de palabra

\begin{tabular}{|l|c|c|c||}
\hline Escolaridad & $\begin{array}{c}\text { Diptongación de la nasal } \\
\text { en coda / } \\
\text { Total de posibilidades }\end{array}$ & Porcentaje & Ejemplos \\
\hline Primero & $290 / 412$ & $70,4 \%$ & ['aß1ãun] \\
\hline Segundo & $246 / 444$ & $55,4 \%$ & [in'tenntãun] \\
\hline Tercero & $188 / 763$ & $24,6 \%$ & ['soun] \\
\hline Cuarto & $305 / 621$ & $49,1 \%$ & ['pideñ] \\
\hline Total & $1.029 / 2.240$ & $46 \%$ & \\
\hline
\end{tabular}

La Tabla 4 indica que, del total de 412 casos de nasal final encontrados en las entrevistas de los alumnos del primer año, hubo 290 casos de diptongación de esta consonante, es decir, el 70,4\%. En los demás casos, hubo 13 ocurrencias de apagamiento de la nasal ${ }^{7} \mathrm{y}$, en los 109 dados restantes, hubo el mantenimiento de la nasal.

En el caso de los estudiantes del segundo año, verificamos que el índice de aplicación del proceso de diptongación ha sido menor: 246 datos (55,4\%). Los demás datos se refieren a la elisión de la nasal -10 casos- y al mantenimiento de esa consonante -188 casos. La muestra referente a las entrevistas del tercer año indica que hubo una disminución significativa en el uso de la diptongación, es decir, 188 datos - el 24,6\%. En los demás casos, la consonante nasal en coda ha sido apagada -10 ocurrencias- o mantenida -565 casos.

En lo referente al cuarto año, hubo un aumento del uso de la diptongación, comparado al tercero. De los 621 casos en que la consonante nasal estaba en coda, hubo 305 ocurrencias del fenómeno investigado, totalizando el 49,1\%. El restante de los datos se refiere a la elisión de la nasal en coda -4 ocurrencias- y a la conservación de la nasal en final de palabra -312 casos.

Cabe tener en cuenta que, en todos los niveles de estudio, en los casos en que hubo la conservación de la nasal también hubo ocurrencias

${ }^{7}$ Ejemplos de elisión de la nasal final: Las personas tienen... [’tjene] / Muchas mujeres tienen... ['tjene]. 
de datos en que la palabra siguiente a la consonante nasal en coda estaba constituida de vocal. ${ }^{8}$ En español cuando una palabra termina en consonante y la siguiente empieza con vocal hay un proceso de resilabeo, por lo tanto, la nasal que está en posición de coda pasa a la posición de ataque. De esa forma, no hay más posibilidad de que esta consonante se diptongue. Ejemplos: ... tuvieron estudio... [tu’ßjerones’tuðjo]; ... hablan español... ['aßlanespa'nol].

Conforme podemos percibir, a través de los resultados de la Tabla 4, los alumnos del primer año del Curso de Letras (Portugués/ Español) de la FURG suelen diptongar la nasal en posición de coda final, lo que genera una interferencia de su Lengua Materna. Por otro lado, hay un menor índice de este proceso en los datos de los alumnos del segundo, tercero y cuarto años del Curso. Conforme mencionamos anteriormente, vale destacar que en la muestra del cuarto año hubo un aumento de la diptongación si la comparamos con la muestra del tercero.

Tabla 5: Sonorización de /s/ intervocálica

\begin{tabular}{|l|c|c|c||}
\hline Escolaridad & $\begin{array}{c}\text { Sonorización de } \\
\text { /s/ intervocálico / } \\
\text { Total de posibilidades }\end{array}$ & Porcentaje & Ejemplos \\
\hline Primero & $28 / 150$ & $18,6 \%$ & [portu'geza] \\
\hline Segundo & $20 / 110$ & $18,2 \%$ & ['fizika] \\
\hline Tercero & $31 / 249$ & $12,4 \%$ & {$[$ 'muzika] } \\
\hline Cuarto & $56 / 172$ & $32,5 \%$ & {$[$ empreza] } \\
\hline Total & $135 / 681$ & $19,8 \%$ & \\
\hline
\end{tabular}

${ }^{8}$ En el primer año de estudio, de los 109 casos de mantenimiento de la nasal, en 41 de ellos la palabra siguiente a esta consonante estaba constituida de una vocal inicial. En el segundo año, de los 188 datos de esta variable, hubo 82 palabras siguientes iniciadas por vocal. En el tercer año, de las 565 ocurrencias de la conservación de la nasal, en 195 el vocablo siguiente estuvo formado por vocal inicial. En el cuarto año, de los 312 casos de aplicación de la forma nativa española, en 48 de ellos la palabra subsecuente a la consonante investigada comenzaba por vocal. 
En la Tabla 5, verificamos que el proceso de sonorización de /s / intervocálica presenta una pequeña incidencia en todos los niveles de estudio, comparado al proceso de diptongación, presentado en la Tabla 4. Con todo, así como en la diptongación y en la vocalización de /1/ en coda, verificamos un aumento de este proceso en la muestra representativa del cuarto año del Curso.

Gráfico 1 - Procesos de transferencia del portugués en el uso del español

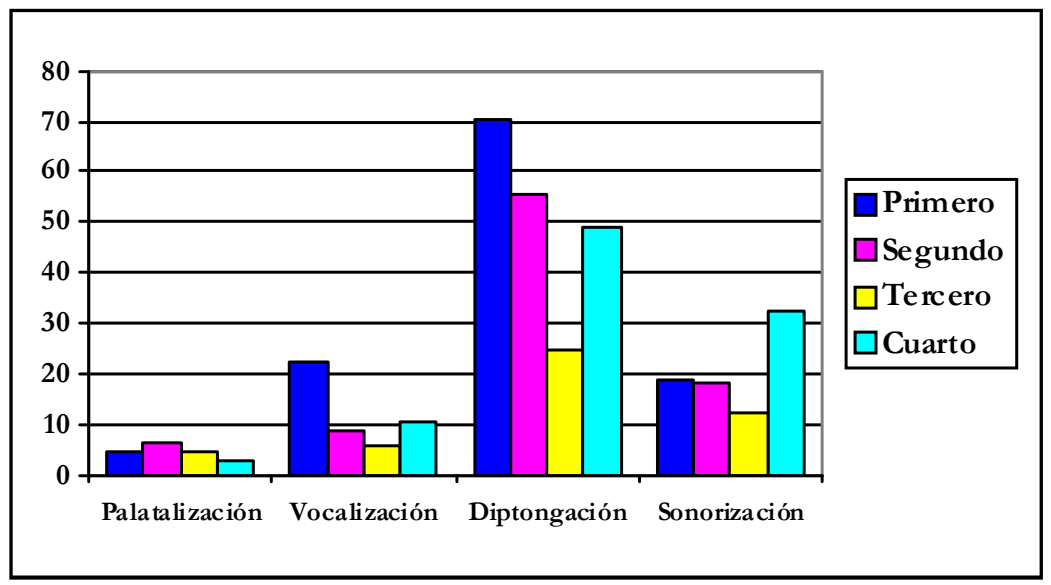

En el Gráfico 1, verificamos los cuatro procesos característicos del portugués que aparecen en el habla de los estudiantes de español de la FURG. Con relación a estos fenómenos, verificamos que la palatalización presenta poca aplicación en todos los niveles de estudio, presentando el 4,8\% de aplicación total.

En lo referente a la vocalización de /1/ en coda, verificamos que a pesar de estar presente en todos los años de escolaridad, presenta un mayor índice en las muestras relativas al primero y al cuarto año, con un $22 \%$ y un $10,5 \%$ de aplicación, respectivamente. Por otro lado, la diptongación de la nasal en final de palabra presenta un alto índice de utilización en todos los años, presentando un porcentaje total de aplicación del 46\%. Evidenciamos una disminución significativa del fenómeno sólo en la muestra relativa al tercer año (el 24,6\%). 
La sonorización de /s/ intervocálica presenta el 19,8\% de aplicación total, mostrando una ligera disminución en los segundos y terceros años, aumentando su surgimiento en el cuarto año.

Vale destacar que los resultados de este estudio son preliminares y que realizaremos nuevas grabaciones, con el fin de acompañar el desarrollo de los académicos de Letras de la FURG en la adquisición del español como lengua extranjera. Sin embargo, los resultados de este estudio, a pesar de no conclusivos, nos auxiliaron a identificar algunas de las principales interferencias fonético/fonológicas de los aprendices, lo que facilitará el modo de actuar en el aula ante tales dificultades.

\section{Conclusiones}

Este artículo trató de investigar la adquisición del sistema consonántico del español por hablantes nativos de portugués, en un ambiente formal de aprendizaje, con el objetivo de verificar si los académicos del Curso de Letras presentan un buen desempeño en la pronunciación de la lengua española. El estudio demostró que los alumnos de todos los niveles de escolaridad presentan algunas interferencias de su Lengua Materna en la Lengua Extranjera.

También observamos que, aunque las interferencias disminuyen con el aumento de tiempo de estudio, ellas permanecen durante los cuatro años del Curso de Letras. Desde nuestro punto de vista, en la enseñanza de español, sea en la Universidad o en las escuelas de Enseñanza Secundaria, es necesario que se empleen constantemente actividades relacionadas a la pronunciación. De ese modo, los profesores obtendrán mejores resultados en el aula.

Vale recordar que el profesor tiene como rol diagnosticar los errores de sus alumnos y aplicar actividades de corrección fonética; para eso, necesita adquirir una formación sólida en fonética y fonología. Sin embargo, no hay que pensar que el profesor de E/LE debe ser un experto en el área, sino que debe poseer nociones fundamentales para que pueda identificar las dificultades de los aprendices y realizar las debidas correcciones de pronunciación.

Una vez que hemos presentado los resultados de nuestra muestra de investigación y hemos mostrado los errores que más frecuentemente 
realizan los alumnos, es necesario sugerir algunas estrategias para intentar corregirlos. A continuación presentamos algunas ideas de actividades que se pueden realizar en el aula, con el fin de hacer que los alumnos superen sus dificultades y de que eviten fosilizar los errores:

a) ejercicios de comprensión auditiva en los que los alumnos deben reconocer sonidos; b) ejercicios con pares mínimos en los que deben escuchar grabaciones para diferenciar sonidos y, también, significados; c) ejercicios de comprensión auditiva en los que deben prestar atención en algún aspecto, ejemplo, en sílabas subrayadas, a fin de percibir como se producen; d) ejercicios de discriminación auditiva, en los que los alumnos deben escuchar y marcar la opción que escuchan; e) ejercicios que hacen referencia a la división silábica, en los que los aprendices deben percibir como se producen, por ejemplo, las consonantes en final de sílaba; f) ejercicios que tratan de la acentuación, en los que identificarán la sílaba tónica de las palabras. En este caso, se pueden crear actividades con palabras heterotónicas en portugués/ español o con vocablos que presentan más de una posibilidad de acento en español, como 'video' y 'vídeo', 'periodo', y 'período'; g) ejercicios con canciones, ya que los alumnos pasan a prestar atención en estructuras, en sonidos e, incluso, en el ritmo de la lengua; h) ejercicios con dictados para estimular al alumno a prestar atención en determinados sonidos del español; i) ejercicios con creación de nuevos trabalenguas, elaborados por los propios alumnos basados en la identificación de alguna dificultad del grupo.

Creemos que a través de esos recursos los estudiantes conseguirán superar sus dificultades, produciendo mensajes comprensibles y correctamente contextualizados en el discurso, siendo capaces de percibir y producir elementos fónicos del español. Además, los profesores conseguirán entender cómo el alumno adquiere los aspectos relativos a la fonología de la lengua meta, facilitando el proceso de aprendizaje.

\section{Bibliografía}

ALARCOS LLORACH, Emilio. Fonología española. 4. ed. Madrid: Gredos, 1991. 
BISOL, Leda. O clítico e seu hospedeiro. Letras de Hoje, Porto Alegre, n. 141, p. 163-184, 2005.

BRANDÃO, Luciana Rodrigues. Yo hablo, pero... ¿quién corrige? A correção e erros fonéticos persistentes nas produções em español de aprendizes brasileiros. Campinas: Unicamp, 2003.

BRISOLARA, L. B., VANDRESEN, P., MATZENAUER, C. L. O prestígio lingüístico como limitador da autonomia En: II Fórum Internacional de Ensino de Línguas Estrangeiras, 2002, Pelotas. Anais do FILE II. Pelotas: EDUCAT, 2002.

CÂMARA JR, Joaquim Mattoso. Estrutura da Lingua Portuguesa. Petrópolis: Vozes, 1970.

CORDER, S.P. The significance of learner's errors. International Review of applied linguistics. t. 1, n. 4, p. 161-170, 1967.

ESPIGA, Jorge. Interferências e interlínguas no aprendizado de Espanhol por falantes nativos de português: aspectos de fonologia. En: MATZENAUER-HERNANDORENA, Carmen Lúcia. Aquisição de Lingua Materna e de Lingua Estrangeira: aspectos fonético-fonológicos. Pelotas: EDUCAT, 2001.

HORA, Dermeval O. A palatalização das oclusivas dentais: variação e representação não-linear. Porto Alegre: EDIPUCRS, 1990.

HORA, Adauto Félix da. Comparación fonológica del español y del portugués de Brasil. Anuario brasileño de estudios hispánicos, 10, p. 15-30, 2000.

MENA-ALVES, Letícia; BRISOLARA, Luciene Bassols. Interferências fonéticas e fonológicas na aquisição de espanhol como língua estrangeira. En: Anais do III Fórum Internacional de Ensino de Linguas Estrangeiras. Pelotas: EDUCAT, 2004.

NAVARRO TOMÁS, Tomás. Manual de pronunciación española. Madrid: Consejo Superior de Investigaciones Científicas, 2004. 
REAL ACADEMIA ESPAÑOLA. Diccionario de la lengua española. Fuente electrónica [en línea], disponible en < http:/ / www.rae.es, acceso el 10/ $02 / 2011>$.

SASSI, María Pía. A palatalização na cidade de Santa Vitória do Palmar. Pelotas: UCPel, 1997. 